\title{
Prevalence and risk factors for mesh erosion after laparoscopic-assisted sacrocolpopexy
}

\author{
Jasmine Tan-Kim • Shawn A. Menefee • \\ Karl M. Luber • Charles W. Nager • Emily S. Lukacz
}

Received: 13 June 2010 / Accepted: 29 August 2010 / Published online: 15 September 2010

(C) The Author(s) 2010. This article is published with open access at Springerlink.com

\begin{abstract}
Introduction and hypothesis The purpose of this study is to identify risk factors for mesh erosion in women undergoing minimally invasive sacrocolpopexy (MISC). We hypothesize that erosion is higher in subjects undergoing concomitant hysterectomy.

Methods This is a retrospective cohort study of women who underwent MISC between November 2004 and January 2009. Demographics, operative techniques, and outcomes were abstracted from medical records. Multivariable regression identified odds of erosion.

Results Of 188 MISC procedures 19(10\%) had erosions. Erosion was higher in those with total vaginal hysterectomy (TVH) compared to both post-hysterectomy ( $23 \%$ vs. $5 \%$, $p=0.003)$ and supracervical hysterectomy (SCH) $(23 \%$ vs. $5 \%, p=0.109$ ) groups. In multivariable regression, the odds
\end{abstract}

Cities in which this study was conducted: La Jolla, CA and San

Diego, CA

J. Tan-Kim · C. W. Nager · E. S. Lukacz

Division of Female Pelvic Medicine and Reconstructive Surgery,

Department of Reproductive Medicine, University of California,

San Diego,

La Jolla, CA, USA

J. Tan-Kim

e-mail: jstan@ucsd.edu

J. Tan-Kim $\cdot$ S. A. Menefee $\cdot$ K. M. Luber

Department of Obstetrics and Gynecology,

Division of Female Pelvic Medicine and Reconstructive Surgery,

Kaiser Permanente San Diego,

San Diego, CA, USA

J. Tan-Kim • E. S. Lukacz $(\bowtie)$

UCSD Women's Pelvic Medicine Center,

9350 Campus Point Dr., Suite 2A,

La Jolla, CA 92037, USA

e-mail: elukacz@ucsd.edu of erosion for TVH was 5.67 (95\% CI: 1.88-17.10) compared to post-hysterectomy. Smoking, the use of collagen-coated mesh, transvaginal dissection, and mesh attachment transvaginally were no longer significant in the multivariable regression model.

Conclusion Based on this study, surgeons should consider supracervical hysterectomy over total vaginal hysterectomy as the procedure of choice in association with MISC unless removal of the cervix is otherwise indicated.

Keywords Erosion · Mesh · Hysterectomy · Laparoscopy · Sacrocolpopexy

\section{Introduction}

Rates of recurrent prolapse after traditional vaginal repairs have been documented as high as $58 \%$ [1] with reoperation rates up to $30 \%$ [2]. The use of synthetic mesh to augment reconstructive procedures has gained popularity over the years in an effort to reduce recurrence. Despite recent trends toward vaginal placement of mesh, mesh has been traditionally used abdominally to correct apical prolapse of the vaginal vault [3]. Sacrocolpopexy was first described by Arthure [4] in 1957 and understanding of this procedure has evolved over the past five decades. A recent comprehensive review of sacrocolpopexy describes the reoperation rate for prolapse of only 2.2\% [5]. Minimally invasive sacrocolpopexy (MISC) has been introduced in an effort to decrease pain, reduce recovery, and improve cosmetic results by eliminating the need for a large abdominal incision [6]. The robotic-assisted laparoscopic sacrocolpopexy (RALSC) and conventional laparoscopic sacrocolpopexy (LSC) have shown similar medium-term efficacy to traditional open approaches with less pain, less blood loss, 
shorter hospital stay, and reduced recovery time [7-9]. For the purposes of this study, the term MISC refers to both the RALSC and LSC.

One complication unique to the use of synthetic mesh is its tendency to erode through adjacent tissue. Erosions are usually defined as visible portions of surgical material, usually mesh or suture, which has become exposed through the epithelium of the vagina or into adjacent visceral organs. Mesh erosion rates with sacrocolpopexy range from $2 \%$ to $10 \%$ in the literature $[5,10-12]$. The consequences of erosions range from negligible to severe.

Modifiable risk factors associated with mesh erosions include smoking [10], concomitant hysterectomy [10], mesh type [13], and placement of mesh transvaginally [14]. The majority of studies assessing mesh erosion have been performed on traditional abdominal sacrocolpopexy (ASC). MISC are performed with a slightly different technique and thus has potentially different risks of erosion. The purpose of this study was to identify rates and risk factors for mesh erosion in women undergoing MISC using various techniques. It is our hypothesis that the mesh erosion rate is higher in subjects undergoing concomitant hysterectomy compared to those women who are post-hysterectomy.

\section{Materials and methods}

This was an IRB-approved, retrospective cohort study of women who underwent MISC using one of two techniques (RALSC or LSC) between November 2004 and January 2009. Surgeries were performed at two institutions responsible for training in the UCSD/Kaiser Permanente San Diego Female Pelvic Medicine and Reconstructive Surgery Fellowship program. Subjects were excluded if they did not have a follow-up postoperative physical examination.

Data was extracted from the electronic medical record and hospital charts. Age, race, gravity, parity, history of diabetes mellitus, smoking, and hormone status along with physical examination including weight and pelvic organ prolapse quantification (POP-Q) [15], was also collected. Operative techniques, complications, blood loss, concomitant procedures, and operative times were abstracted from the hospital records. Postoperative follow-up visits including POP-Q and the detection of a mesh erosion were also documented.

\section{Operative techniques}

The RALSC technique was performed exclusively at UCSD by two attending surgeons. If a hysterectomy was indicated, a laparoscopic supracervical hysterectomy $(\mathrm{SCH})$ was performed in a routine fashion with the uterine corpus removed using a morcellator. The da Vinci robot-S (Intuitive
Surgical Inc., Sunnyvale, CA) was docked to three of the operative ports and a camera port. The vesicovaginal, rectovaginal, and presacral spaces were all dissected with robotic assistance. The vaginal dissections were performed with the assistance of a vaginal probe to delineate the planes of dissection. In all cases, a non-coated type 1 monofilament polypropylene mesh was attached to the anterior and posterior vaginal walls (and cervical stump if applicable) with a minimum of four monofilament permanent sutures to each vaginal wall. Two permanent sutures were placed at the level of the sacral promontory to secure the mesh to the anterior longitudinal ligament. The peritoneum was then reapproximated over the mesh with absorbable suture.

The LSC technique was performed exclusively at Kaiser Permanente by four attending surgeons using three techniques: (1) entire procedure performed laparoscopically for the subjects who were post-hysterectomy, (2) total vaginal hysterectomy (TVH) with mesh attachment transvaginally prior to laparoscopic portion of the procedure (vaginally assisted laparoscopic sacrocolpopexy (VALSC)), and (3) TVH followed by mesh attachment laparoscopically after vaginal closure of the cuff (vaginal hysterectomy prior to laparoscopic sacrocolpopexy (VHLSC)). One of the two types of mesh were used for each procedure; a type 1 monofilament polypropylene mesh coated with a hydrophilic porcine collagen (Pelvitex, Bard, Covington, GA) or noncoated type 1 monofilament polypropylene. For the laparoscopic portion of each case, a total of four to five ports were placed. For subjects who were post-hysterectomy prior to this procedure, the spaces were dissected laparoscopically with the use of a vaginal probe. Laparoscopic attachment of mesh took place using a minimum of four polypropylene monofilament sutures to each wall. If a hysterectomy was indicated, a total vaginal hysterectomy was performed followed by transvaginal development and dissection of the anterior and posterior vaginal planes in most cases. For the VALSC group, mesh was attached transvaginally to the anterior and posterior vaginal surfaces with at least four polypropylene monofilament sutures. The tails of the mesh were then placed into the intraperitoneal cavity followed by transvaginal closure of the cuff. The laparoscopic attachment of the mesh to the anterior longitudinal ligament followed. Alternatively, the VHLSC group underwent TVH and dissection followed by closure of the vaginal cuff. Mesh placement and attachment was then performed laparoscopically.

For all LSC cases, the proximal tails of the mesh were attached to the anterior longitudinal ligament at the level of the sacral promontory using two to four titanium sutures (Protack; Tyco Healthcare, Norwalk, CT) or interrupted monofilament permanent suture. The peritoneum was reapproximated over the mesh with absorbable suture.

For all MISC, concomitant repairs such as anterior repairs, posterior repairs, or mid-urethral slings were performed either 
prior to the sacrocolpopexy or after. The variations in technique for all procedures or the type of mesh used were based on surgeon preference.

All included subjects were evaluated postoperatively at standard intervals. They had a POP-Q examination, vaginal inspection with a clear plastic speculum, and digital rectal examination to evaluate prolapse status and presence of mesh erosion. Our primary outcome of erosion was identified if any mesh or permanent suture material was seen in the vagina or other adjacent tissues.

Chi-square and Fisher exact tests were used to compare categorical variables and compare erosion rates between various groups. The Mann Whitney U and Wilcoxon's rank tests were used to compare non-parametric data and explore variables which may represent risk factors for mesh erosion. Multivariable logistic regression was used to assess independent predictors for mesh erosion while controlling for potential confounders. Variables were included if they had a $p$ value $<0.10$ on univariate analysis. Odd ratios (OR) and 95\% confidence intervals (CI) are reported. A $p$ value of $<0.05$ was considered statistically significant. Statistical analysis was performed with PASW 18 (SPSS, Inc, Chicago, IL).

Based on a study of ASC by Thompson et al., subjects who had previous hysterectomy had a mesh erosion rate of $0.7 \%$, while those who underwent concomitant total abdominal hysterectomy had an erosion rate of $13.6 \%$ [16]. We based our power analysis on this data which indicated that 50 subjects would be required in each group in order to detect a difference in mesh erosion rate of $1 \%$ to $14 \%$ with $\alpha=0.05$ and $80 \%$ power.

\section{Results}

A total of 196 women underwent MISC and 96\% had sufficient data for follow-up. The mean age of the 188 women was $61 \pm 9$ years and median prolapse stage was 3 (2-4). Median follow-up was 20 weeks (3-124 weeks) in the RALSC compared to 14 weeks (2-171 weeks) for LSC group ( $p=0.280$ ). All 21 women with concomitant SCH had RALSC and all 57 women with concomitant TVH had LSC. Of those who had a TVH, 29 had the mesh attached to the vagina transvaginally (VALSC) while 28 had the mesh attached laparoscopically (VHLSC). The overall mesh erosion rate was $10 \%(19 / 188)$. Mesh erosion rates were $5 \%$ in both the post-hysterectomy and $\mathrm{SCH}$ groups $(p=0.984)$. In the concomitant TVH group, mesh erosion rates were $23 \%(p=0.003)$. A subanalysis was performed on the subjects with TVH who were divided into VALSC and VHLSC. VHLSC had a higher erosion rate than VALSC, although this difference was not statistically significant (Fig. 1).

Of the 19 erosions, 1 involved suture only, 15 involved exposed mesh at the level of the apex, 1 occurred along the anterior wall, 1 resulted in a vesicovaginal fistula and 1 erosion was into the bladder without fistula. There were no erosions noted at the level of a mid-urethral
Fig. 1 Mesh erosion rates in women undergoing minimally invasive sacrocolpopexy (MISC) using various techniques. $\mathrm{PH}$ post-hysterectomy (reference group), $\mathrm{SCH}$ supracervical hysterectomy, TVH total vaginal hysterectomy, VALSC vaginally assisted laparoscopic sacrocolpopexy (transvaginal placement of mesh), and VHLSC vaginal hysterectomy prior to laparoscopic sacrocolpopexy (laparoscopic placement of mesh). Asterisk Fisher's exact test

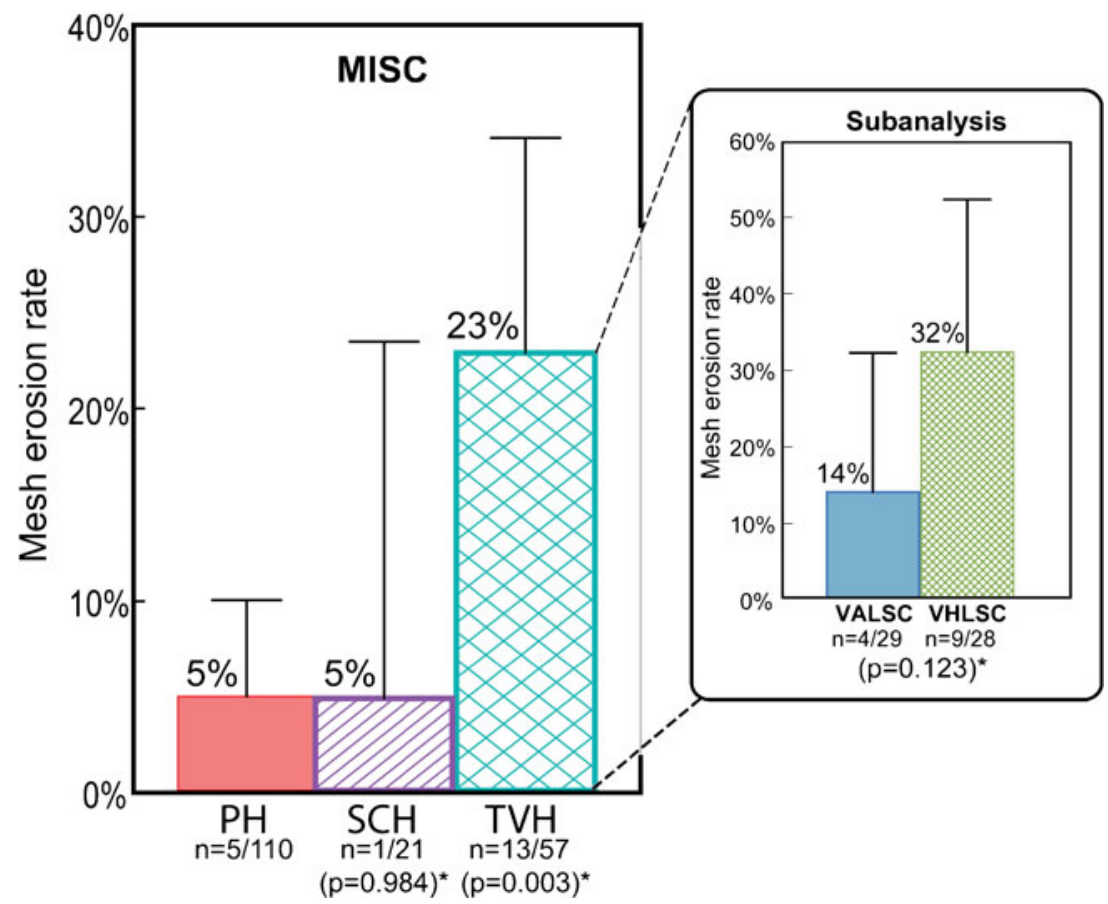


sling or along the posterior wall of the vagina. The mean time to mesh erosion detection was $23 \pm 21$ weeks (range 3-86 weeks) with a median of 14 weeks (all 19 subjects). There was only one subject in the SCH group with mesh erosion which occurred at 6 week. The mean time to erosion for the post-hysterectomy subjects were $31 \pm 29$ weeks (median 27, $n=5$ ) and 21 \pm 17 weeks (median 12, $n=13$ ) for the TVH subjects, this difference did not meet statistical significance $(p=0.661)$.

There were no differences between subjects who did and did not develop mesh erosion except for concurrent posterior colporrhaphy and hysterectomy $(p<0.1$ (Table 1$)$ ). Multivariable regression revealed that only concurrent TVH remained a significant contributor to mesh erosion (Table 2). Concurrent TVH was performed in 30\% (57/188) of subjects and was associated with a nearly sixfold increase in erosion compared to post-hysterectomy. The odds of mesh erosion with supracervical hysterectomy were equal to post-hysterectomy.

A subanalysis was performed using three variables potentially increasing the risk of mesh erosion in subjects undergoing TVH. A multivariate logistic regression model was performed on the (1) utilization of transvaginal dissection $(n=53)$, (2) use of a collagen-coated polypropylene mesh $(n=46)$ and (3) attachment of mesh transvaginally (VALSC, $n=29$ ) or laparoscopically (VHLSC, $n=28$ ) and (4) attending surgeon. None of these variables were found to be significant to the contribution of mesh erosion (data not shown). There was a trend towards increased mesh erosion with mesh placed laparoscopically as opposed to transvaginally (OR 2.96; 95\%CI: 0.78-11.3) although this finding was not statistically significant.
Table 1 Univariate analysis of demographics, baseline characteristics, and concomitant procedures
HRT Hormone replacement therapy - systemic, $\mathrm{Hgb}$ Hemoglobin $\mathrm{SCH}$ Supracervical hysterectomy, TVH Total vaginal hysterectomy

${ }^{\text {a }}$ Continuous data summarized as mean $\pm \mathrm{SD}$ and compared between groups with the use of MannWhitney $U$ test $(<30$ cases, central limit theorem)

${ }^{\mathrm{b}}$ Non-parametric continuous data summarized as mean (median) and compared with the use of the Wilcoxon's rank test

${ }^{\mathrm{c}}$ Categorical data summarized as frequency (\%) and compared between groups with the use of the $X^{2}$ test

${ }^{\mathrm{d}}$ Fisher's Exact test

\begin{tabular}{|c|c|c|c|}
\hline Variable & No mesh erosion $(n=169)$ & $\operatorname{Mesh} \operatorname{erosion}(n=19)$ & $p$ value \\
\hline Age (years) & $60.7 \pm 9.2$ & $61.4 \pm 5.9$ & $0.707^{\mathrm{a}}$ \\
\hline Gravity & $3.4(3)$ & $2.8(3)$ & $0.940^{\mathrm{b}}$ \\
\hline Para & $2.9(3)$ & $2.5(3)$ & $0.695^{\mathrm{b}}$ \\
\hline Weight (lbs) & $155.2 \pm 24.8$ & $147.2 \pm 24.8$ & $0.245^{\mathrm{a}}$ \\
\hline Race & & & $0.912^{\mathrm{c}}$ \\
\hline White & $80 \%$ & $74 \%$ & \\
\hline Hispanic & $15 \%$ & $21 \%$ & \\
\hline Asian & $4 \%$ & $5 \%$ & \\
\hline AA & $1 \%$ & $0 \%$ & \\
\hline Other & $1 \%$ & $0 \%$ & \\
\hline Diabetes mellitus & $9 \%$ & $16 \%$ & $0.401^{\mathrm{d}}$ \\
\hline Smoking & & & $0.341^{\mathrm{c}}$ \\
\hline Current & $4 \%$ & $5 \%$ & \\
\hline Past & $18 \%$ & $32 \%$ & \\
\hline HRT/menopause & & & $0.394^{\mathrm{c}}$ \\
\hline Premenopausal & $17 \%$ & $5 \%$ & \\
\hline Menopause+HRT & $27 \%$ & $26 \%$ & \\
\hline Menopause no HRT & $56 \%$ & $68 \%$ & \\
\hline Preop Ba & $2.9(3)$ & $2.9(3)$ & $0.391^{\mathrm{b}}$ \\
\hline Preop C & $1.0(1)$ & $0.4(0)$ & $0.562^{\mathrm{b}}$ \\
\hline Preop Bp & $0.1(-1)$ & $-0.05(0)$ & $0.387^{\mathrm{b}}$ \\
\hline Prolapse stage & $2.8(3)$ & $2.9(3)$ & $0.558^{\mathrm{b}}$ \\
\hline Postop Hgb & $10.9 \pm 1.2$ & $10.9 \pm 0.8$ & $0.901^{\mathrm{a}}$ \\
\hline Surgery time (min) & $255.7 \pm 71.0$ & $262.3 \pm 67.8$ & $0.512^{\mathrm{a}}$ \\
\hline Anterior repair & $2 \%$ & $5 \%$ & $0.349^{\mathrm{d}}$ \\
\hline Paravaginal repair & $9 \%$ & $16 \%$ & $0.401^{\mathrm{d}}$ \\
\hline Posterior repair & $15 \%$ & $32 \%$ & $0.075^{\mathrm{c}}$ \\
\hline Mid-urethral sling & $46 \%$ & $47 \%$ & $0.920^{\mathrm{c}}$ \\
\hline Hysterectomy status & & & $0.001^{\mathrm{c}}$ \\
\hline Post-hysterectomy & $62 \%$ & (5/19) $26 \%$ & \\
\hline $\mathrm{SCH}$ & $12 \%$ & (1/19) $5 \%$ & \\
\hline TVH & $26 \%$ & (13/19) 68\% & \\
\hline
\end{tabular}


Table 2 Multivariable logistic regression model for posterior repair and type of hysterectomy

${ }^{a}$ Reference group

$S C H$ supracervical hysterectomy, $T V H$ total vaginal hysterectomy

Of the 19 erosions, 15 were initially treated with vaginal estrogen. Only three (20\%) resolved with estrogen therapy. A total of $10(53 \%)$ required additional surgical procedures while five (26\%) opted for expectant management (Fig. 2). The decision to proceed with surgical management was based on subject's preference, symptoms, or the severity of the mesh erosion. Subject no. 1 had transvaginal resection of exposed mesh with repair of incidental cystotomy which occurred on the posterior bladder wall at the time of mesh resection. Subject no. 2 had a vesicovaginal fistula with erosion above the trigone, which was repaired after two separate procedures, the second procedure included a laparotomy with omental J-flap. Subject no. 3 had a suture excised transvesically at the time of initial operation, she subsequently developed $<1 \mathrm{~cm}$ portion of mesh in her bladder and declined surgical excision. She has been expectantly managed with periodic cystoscopy since March 2007 and continues to have a stable erosion without evidence of inflammation or recurrent infections.

\section{Discussion}

Mesh erosion associated with pelvic reconstructive surgery is a recognized complication of using synthetic mesh. Erosions may be asymptomatic and inconsequential or they may present with severe infection or result in fistulae.
Identification of modifiable risk factors for the development of this complication is critical. Our study demonstrates a sixfold increase risk of mesh erosion when TVH is performed at the time of MISC compared to subjects who had MISC post-hysterectomy or with SCH. After careful analysis of all potentially contributing variables, only the TVH remained a significant modifiable risk factor. Only $20 \%$ of those with erosions responded to conservative estrogen therapy alone.

Our findings are supported by studies of total abdominal hysterectomy (TAH) and open ASC. Cundiff et al. found that TAH at the time of ASC resulted in a rate of erosion of $14 \%$, which was a fivefold increase risk over subjects who had ASC without hysterectomy [10]. Bensinger et al. showed a sevenfold increase risk of mesh erosion with subjects who had a TAH at the time of their ASC compared with subjects who underwent $\mathrm{SCH}$ at the time of ASC [17]. Despite differences in operative techniques for MISC, there appears to be comparable erosion rates. Our study is the first to compare erosions in MISC in a large cohort of women.

Mesh erosion may result from a combination of bacterial infection and devascularization of the vaginal cuff. Opening of the vaginal cuff with exposure of the surgical bed to vaginal flora may be a key component in the evolution of subsequent erosion. Our reported mesh erosion rate of $23 \%$ for subjects who had concomitant TVH with MISC is
Fig. 2 Only $3(20 \%)$ of the 15 erosions resolved with estrogen therapy alone. A total of 10 $(53 \%)$ of the 19 erosions required surgical management for definitive treatment and all 10 of these erosions resolved after surgery. Of the 19 subjects, five $(3+2 ; 26 \%)$ ultimately opted for expectant management without further follow-up at the time of this review

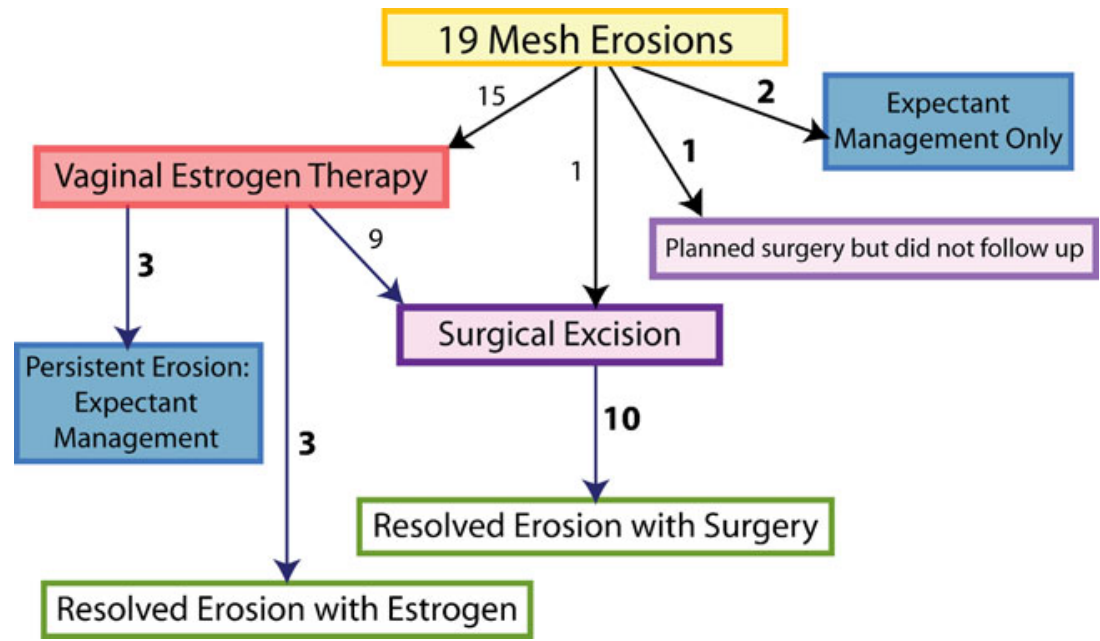


higher than rates reported in the literature for TAH with ASC. Our erosion rates were also significantly higher than those reported by a large retrospective study in which laparoscopic-assisted vaginal hysterectomies (LAVH) were performed with laparoscopic sacrocolpopexy [18]. There are several possible explanations for this finding. TVH inherently differs from TAH, TLH, and even LAVH in the amount of surgical bed exposure to vaginal flora which may result in higher risk of infection. This is supported by the observation that subjects who underwent a supracervical hysterectomy at the time of their sacrocolpopexy had the same rate of erosion as the post-hysterectomy group. Our overall rates of mesh erosion for post-hysterectomy subjects $(5 \%)$ and those with concomitant SCH $(5 \%)$ are consistent with the current literature $[10,19]$. This finding supports the adoption of SCH over TVH when a hysterectomy is indicated unless removal of the cervix is clinically necessary. However, while SCH may be associated with lower erosion rates, there may be other consequences such as cervical elongation, subsequent development of dysplasia, or bleeding problems that should be balanced against the risk of erosion.

The recent introduction of minimally invasive techniques has improved recovery times, decreased length of hospital stay, decreased intraoperative blood loss, improved postoperative pain, and reduced time to resume normal activity $[9,20-23]$. Unfortunately, these techniques tend to take longer to perform $[9,24]$. Surgeons continue to modify their techniques in order to decrease operative time which decreases operative morbidity. Large retrospective studies demonstrated low mesh erosion rates with LAVH and transvaginal cuff closure $(2.3 \%)$ which prompted our institution to develop the VALSC and VHLSC technique to reduce surgical duration [18]. Combined abdominal-vaginal procedures have been previously described. One small study reported a $40 \%$ mesh erosion rate but included only five cases [14] while a larger retrospective study of 169 patients who underwent abdominovaginal sacral colpoperineopexy had a documented mesh erosion rate of $6.5 \%$ with a median time to erosion of 3 months [25]. A subanalysis of our data revealed that concomitant TVH was the single contributing factor for mesh erosion, not the performance of transvaginal dissections. In addition, contrary to what was expected, we found an increased risk of erosion when the mesh was placed laparoscopically as opposed to transvaginally after $\mathrm{TVH}$, but this difference was not statistically significant.

It has been documented that mesh properties play a role in the relative risk of developing mesh erosion. Collagencoated polypropylene mesh was used at our institution after several reports indicated prevention of erosion by positioning a collagen barrier between the synthetic mesh and vaginal mucosa. One study included 29 subjects and no mesh erosions were observed over a 2-year period [26].
However, this study was conducted in post-hysterectomy subjects only and therefore did not result in any inoculation of the collagen-coated mesh with vaginal flora. In vivo animal studies have observed slightly higher levels of acute inflammation with the collagen-coated mesh when compared to non-coated mesh [27]. Another study confirmed the colonization of collagen-coated mesh with greater than 14 different types of bacteria when placed vaginally [28]. Although we did not find a statistically significant difference in mesh erosion between the collagen-coated and non-coated material there was a trend towards increased erosion in the collagen-coated group (OR: 3.53; 95\% CI:0.40-31.4).

The mesh erosions in our cohort were managed both conservatively and surgically. For $20 \%$ of our subjects, estrogen alone was sufficient to resolve their mesh erosion which is similar to other reports of $18.5 \%$ of cases treated successfully with vaginal estrogen or antibiotic cream [29]. One subject was treated successfully with office excision. In $47 \%$ of our subjects, conservative management or office resections were unsuccessful and they chose to return to the operating room for a mesh excision. There were no recurrences of erosion after surgical excision. The remaining 32\% either had persistent erosions which were asymptomatic and declined further treatment or they did not have further follow-up. Cundiff et al. reported in their study that 13 of 17 mesh erosions required at least one surgical intervention [10]. Other studies have shown that definitive surgical excision was ultimately required in $80-100 \%$ of cases $[14,29]$. In our study, a total of seven subjects had some form of bladder integrity compromise at the time of their LSC; two of these subjects went on to have mesh erosions involving the bladder. This suggests that the usage of mesh should be minimized in areas where bladder disruption has occurred intraoperatively.

Limitations of this study are primarily related to its retrospective nature. We found a statistically significant increased risk of mesh erosion with $\mathrm{TVH}$, but our relatively small sample size results in a wide confidence interval which may impact the clinical relevance of this finding. Our study was likely underpowered to detect a difference between transvaginal dissections of the planes, the usage of collagen-coated mesh, or the approach of mesh placement. This study took place at two institutions with six different surgeons performing different procedures. Neither of the institutions performed total laparoscopic hysterectomies at the time of sacrocolpopexy which limits the ability of this study to make conclusions with regard to this technique of minimally invasive hysterectomy. Follow-up intervals were not standard between all subjects, so it is possible that with time, the mesh erosion rates would gradually increase and certain risk factors which were not found to be significant would actually be associated with mesh erosion if the study was prolonged. In some cases our follow-up duration was 
relatively short making it conceivable that the mesh erosion rate is actually underestimated in this population. The retrospective nature of this study made it difficult to assess subjective symptoms of mesh erosion or resolution therefore we only reported on our objective findings. We also did not assess or report on any data related to sexual dysfunction, urinary incontinence, defecatory dysfunction, or pain. We also did not find a significant relationship between mesh erosion and smoking as has been reported in other studies $[10,29]$. This is likely due to the small number of subjects who gave a positive history of smoking $(<4 \%)$. Our primary objective was to assess the prevalence and risk factors associated with mesh erosion. The strengths of this study include the evaluation of two types of MISC and the inclusion of both TVH and SCH. Additionally, because of the nature of the UCSD and Kaiser Permanente Health Care systems, we are confident that subjects who develop erosions would return to our institutions for management.

In conclusion, post-hysterectomy MISC and MISC associated with concomitant $\mathrm{SCH}$ have acceptably low rates of mesh erosions. However concomitant TVH at the time of MISC results in a high rate of mesh erosion with a sixfold increased risk. Based on these data surgeons should consider $\mathrm{SCH}$ as the procedure of choice in association with MISC unless removal of the cervix is otherwise indicated.

Acknowledgements We would like to thank Dr. Kenneth C. Su and Dr. Margie A. Kahn for their help with this study.

Funding No source of funding was used for this study.

Conflicts of interest Drs. Nager and Lukacz are consultants for Intuitive Surgical, Inc.

Open Access This article is distributed under the terms of the Creative Commons Attribution Noncommercial License which permits any noncommercial use, distribution, and reproduction in any medium, provided the original author(s) and source are credited.

\section{References}

1. Whiteside JL, Weber AM, Meyn LA, Walters MD (2004) Risk factors for prolapse recurrence after vaginal repair. Am J Obstet Gynecol 191(5):1533-1538

2. Olsen AL, Smith VJ, Bergstrom JO, Colling JC, Clark AL (1997) Epidemiology of surgically managed pelvic organ prolapse and urinary incontinence. Obstet Gynecol 89(4):501-506

3. Maher CF, Qatawneh AM, Dwyer PL, Carey MP, Cornish A, Schluter PJ (2004) Abdominal sacral colpopexy or vaginal sacrospinous colpopexy for vaginal vault prolapse: a prospective randomized study. Am J Obstet Gynecol 190(1):20-26

4. Arthure HG, Savage D (1957) Uterine prolapse and prolapse of the vaginal vault treated by sacral hysteropexy. J Obstet Gynaecol Br Emp 64(3):355-360
5. Diwadkar GB, Barber MD, Feiner B, Maher C, Jelovsek JE (2009) Complication and reoperation rates after apical vaginal prolapse surgical repair: a systematic review. Obstet Gynecol 113 (2 Pt 1):367-373

6. Maher C, Baessler K, Glazener CM, Adams EJ, Hagen S (2007) Surgical management of pelvic organ prolapse in women. Cochrane Database Syst Rev. doi:10.1002/14651858.CD004014. pub3, Issue 3. Art. No.: CD004014

7. Ganatra AM, Rozet F, Sanchez-Salas R, Barret E, Galiano M, Cathelineau $X$ et al (2009) The current status of laparoscopic sacrocolpopexy: a review. Eur Urol 55:1089-1105

8. Kramer BA, Whelan CM, Powell TM, Schwartz BF (2009) Robot-assisted laparoscopic sacrocolpopexy as management for pelvic organ prolapse. J Endourol 23(4):655-658

9. Geller EJ, Siddiqui NY, Wu JM, Visco AG (2008) Short-term outcomes of robotic sacrocolpopexy compared with abdominal sacrocolpopexy. Obstet Gynecol 112(6):1201-1206

10. Cundiff GW, Varner E, Visco AG, Zyczynski HM, Nager CW, Norton PA et al (2008) Risk factors for mesh/suture erosion following sacral colpopexy. Am J Obstet Gynecol 199(6):688.e1688.e5

11. Kohli N, Walsh PM, Roat TW, Karram MM (1998) Mesh erosion after abdominal sacrocolpopexy. Obstet Gynecol 92(6): 999-1004

12. Feiner B, Jelovsek JE, Maher C (2009) Efficacy and safety of transvaginal mesh kits in the treatment of prolapse of the vaginal apex: a systematic review. BJOG 116(1):15-24

13. Begley JS, Kupferman SP, Kuznetsov DD, Kobashi KC, Govier $\mathrm{FE}, \mathrm{McGonigle} \mathrm{KF}$ et al (2005) Incidence and management of abdominal sacrocolpopexy mesh erosions. Am J Obstet Gynecol 192(6):1956-1962

14. Visco AG, Weidner AC, Barber MD, Myers ER, Cundiff GW, Bump RC et al (2001) Vaginal mesh erosion after abdominal sacral colpopexy. Am J Obstet Gynecol 184(3):297-302

15. Bump RC, Mattiasson A, Bo K, Brubaker LP, DeLancey JO, Klarskov P et al (1996) The standardization of terminology of female pelvic organ prolapse and pelvic floor dysfunction. Am J Obstet Gynecol 175(1):10-17

16. Thompson PK, Pugmire JE, Sangi-Haghpeykar H (2004) Abdominal sacrocolpopexy utilizing gore-tex in genital prolapse: unresolved issues. J Pelvic Med Surg 10(6):311-317

17. Bensinger G, Lind L, Lesser M, Guess M, Winkler HA (2005) Abdominal sacral suspensions: analysis of complications using permanent mesh. Am J Obstet Gynecol 193(6):2094-2098

18. Stepanian AA, Miklos JR, Moore RD, Mattox TF (2008) Risk of mesh extrusion and other mesh-related complications after laparoscopic sacral colpopexy with or without concurrent laparoscopic-assisted vaginal hysterectomy: experience of 402 patients. J Minim Invasive Gynecol 15(2):188-196

19. Sarlos D, Brandner S, Kots L, Gygax N, Schaer G (2008) Laparoscopic sacrocolpopexy for uterine and post-hysterectomy prolapse: anatomical results, quality of life and perioperative outcome-a prospective study with 101 cases. Int Urogynecol J Pelvic Floor Dysfunct 19(10):1415-1422

20. Ross JW (1996) Laparoscopic approach for severe pelvic vault prolapse. J Am Assoc Gynecol Laparosc 3(4, Supplement):S43

21. Ross JW (1997) Techniques of laparoscopic repair of total vault eversion after hysterectomy. J Am Assoc Gynecol Laparosc 4 (2):173-183

22. Ross JW, Preston M (2005) Laparoscopic sacrocolpopexy for severe vaginal vault prolapse: five-year outcome. J Minim Invasive Gynecol 12(3):221-226

23. Klauschie JL, Suozzi BA, O'Brien MM, McBride AW (2009) A comparison of laparoscopic and abdominal sacral colpopexy: objective outcome and perioperative differences. Int Urogynecol J Pelvic Floor Dysfunct 20(3):273-279 
24. Paraiso MF, Walters MD, Rackley RR, Melek S, Hugney C (2005) Laparoscopic and abdominal sacral colpopexies: a comparative cohort study. Am J Obstet Gynecol 192(5):17521758

25. Su KC, Mutone MF, Terry CL, Hale DS (2007) Abdominovaginal sacral colpoperineopexy: patient perceptions, anatomical outcomes, and graft erosions. Int Urogynecol J Pelvic Floor Dysfunct 18(5):503-511

26. Ross JW (2007) The use of a xenogenic barrier to prevent mesh erosion with laparoscopic sacrocolpopexy. J Minim Invasive Gynecol 14(4):470-474
27. de Tayrac R, Alves A, Therin M (2007) Collagen-coated vs noncoated low-weight polypropylene meshes in a sheep model for vaginal surgery. A pilot study. Int Urogynecol J Pelvic Floor Dysfunct 18(5):513-520

28. Vollebregt A, Troelstra A, van der Vaart CH (2009) Bacterial colonisation of collagen-coated polypropylene vaginal mesh: are additional intraoperative sterility procedures useful? Int Urogynecol J Pelvic Floor Dysfunct 20(11):1345-1351

29. Lowman JK, Woodman PJ, Nosti PA, Bump RC, Terry CL, Hale DS (2008) Tobacco use is a risk factor for mesh erosion after abdominal sacral colpoperineopexy. Am J Obstet Gynecol 198(5):561.e1-561.e4 